\title{
Del trabajo como certeza al trabajo incertidumbre
}

\author{
From work as certainty to work as uncertainty
}

María Cristina Valencia Cepeda ${ }^{1}$

\section{Resumen}

El capitalismo posindustrial, la automatización y las nuevas condiciones laborales cambian el adjetivo del trabajo como certeza establecido en el capitalismo industrial y se abre paso al trabajo incertidumbre, donde nuevas dinámicas en el mundo laboral reconfiguran el bienestar que parecía asegurado al obtener un empleo. El objetivo de este ensayo es reflexionar sobre cómo las reglas del juego del capitalismo neoliberal pusieron en el centro de la provisión de bienestar al mercado y determinaron cambios en el mundo del trabajo, dejando en un papel secundario al Estado que define sus respuestas de programas sociales situando al individuo como responsable de su trayectoria, sin considerar las variables contextuales, exacerbando la incertidumbre. Finalmente, se analizan propuestas de gestión de esa incertidumbre desde un Estado activo, como el ingreso básico universal y respuestas alternativas desde la comunidad, en la economía social, pues frente a esta reconfiguración del mundo que da lugar a amenazas sin precedentes, deben surgir oportunidades sin precedentes que permitan gestionar la incertidumbre.

Palabras claves: Mercado de trabajo, Condiciones de empleo, Programa social, Bienestar social

\section{Abstract}

Post-industrial capitalism, automation and the new working conditions change the adjective of work as certainty established in industrial capitalism and work opens its way to uncertainty, where new dynamics in the world of work reconfigure the well-being that seemed assured when obtaining a job. The objective of this essay is to reflect on how

Recibido: 6 de septiembre de $2021 \sim$ Aceptado: 6 de enero de 2022 Publicado: 7 de enero de 2022

${ }^{1}$ Psicóloga Clínica de la Pontificia Universidad Católica del Ecuador, Máster en Investigación e Intervención Psicosocial de la Universitat Autónoma de Barcelona, candidata a Magíster en Diseño y Gestión de Programas Sociales de la Facultad Latinoamericana de Ciencias Sociales- Sede Argentina. Docente, Universidad Técnica de Ambato, Ambato, Ecuador. Correo electrónico: cvalenciacepeda@yahoo.com.mx ID http://orcid.org/00000003-2264-0976 
the rules of the game of neoliberal capitalism put the market at the center of the provision of welfare and determined changes in the world of work, leaving in a secondary role the State that defines its responses in social programs placing the individual as responsible for their trajectory, without considering contextual variables, exacerbating uncertainty. Finally, proposals for managing this uncertainty are analyzed from an active State, such as universal basic income and alternative responses from the community, from the social economy, because in the face of this reconfiguration of the world that gives rise to unprecedented threats, opportunities must arise without precedents to manage uncertainty.

Keywords: Labour market, Conditions of employment, Social programmes, Social welfare

\section{Cambios en el mercado de trabajo}

El trabajo como elemento constitutivo de la sociedad y constituyente del sujeto, que da esperanza e identidad, se modifica con la instalación de políticas neoliberales que minan el adjetivo de seguridad que se le otorgaba al trabajo. De modo que, el trabajo sigue siendo central, pero se pasa del trabajo certeza al trabajo incertidumbre, surgen nuevos riesgos, nuevos miedos y la esperanza queda atrás, entonces, ¿qué hacer cuando el miedo sobrepasa a la esperanza? Este enfrentamiento entre los adjetivos del trabajo y la gestión de estos nuevos riesgos son los temas que se plantean en el análisis a continuación y se presentan los cambios en el mercado de trabajo como: la automatización, la aparición de la flexibilidad laboral y la dinámica de los sindicatos.

Desde los albores de la civilización, el alto costo de mover bienes, ideas y personas determinó las formas de producción, la dinámica de comercio y consumo. A medida que avanzó la tecnología, los tres costos disminuyeron, las denominadas TIC redujeron el costo de mover ideas. Hasta la década de 1980 las fábricas y distritos industriales fueron prominentes, pero con la nueva globalización de la tecnología a partir de 1990 se dan procesos de deslocalización, lo que impacta en la automatización de los trabajos industriales. Se empieza a hablar del fin del empleo en la fabricación masiva (Smith, 2021). En todas las economías avanzadas, la proporción de puestos de trabajo en la industria manufacturera disminuye progresivamente, la participación del empleo en los EEUU cayó del 30 por ciento en la década de 1970 al 10 por ciento en 2010. El Reino Unido, que solía absorber más de un tercio de los trabajadores, ahora cuenta con solo 
uno de cada diez trabajos (Baldwin, 2019). En América Latina es especialmente importante analizar este fenómeno pues tiene un mayor porcentaje de empleos susceptibles a la automatización tecnológica (Montané \& Sartorio, 2018).

Una nueva fase de avance tecnológico se da desde 2017 cuando las computadoras son tan buenas o mejores que los humanos en algunas tareas cognitivas. El resultado de esta fase de automatización es que ahora afecta a los trabajos de oficina y no solo los trabajos de fábrica como en el pasado (Acemoğlu, 2021). La automatización que consiste en usar máquinas y algoritmos para realizar tareas que antes se hacían con fuerza de trabajo, no es algo nuevo, pero en el pasado la automatización era solo un aspecto de una amplia plataforma tecnológica y su posible incidencia negativa en el empleo estaba contrarrestada por otras tecnologías. Sin embargo, eso ya no sucede, la unión entre aprendizaje automático e inteligencia artificial abre paso a una nueva fase de automatización (Acemoğlu, 2021). Pues si bien los efectos de la automatización sobre la economía, el empleo y sobre los trabajadores ha sido una preocupación desde el siglo XVIII, en el siglo XXI, la discusión sobre el desempleo tecnológico se ha vuelto a reavivar a causa de los avances en robótica y tecnologías de la información y la comunicación (Arguello Verbanaz, 2019). En 2020 se estima que dos tercios de las empresas estaban aumentando la inversión en automatización e inteligencia artificial de manera significativa; esto implica que hasta un 25 por ciento más de trabajadores de lo que se había estimado están potencialmente necesitando cambiar de ocupación (McKinsey Global Institute, 2021b). Precisamente, uno de los múltiples efectos de la pandemia sobre el mercado laboral es la aceleración de los procesos de automatización en las empresas (Ubieto, 2021), las restricciones, sin duda, han dado a los empleadores más razones para tratar de reemplazar a los trabajadores con máquinas (Acemoğlu, 2021).

En Latinoamérica la tendencia a la automatización de las empresas tiene una especial implicación, pues las empresas latinoamericanas a menudo han quedado rezagadas con respecto a los países más desarrollados en cuanto a la adopción de nuevas tecnologías, por lo que parece un obstáculo para que la automatización crezca rápidamente, pudiendo generar un nuevo tipo de brecha digital internacional (Hewitt \& Monge-González, 2018). Además, en el contexto global de relocalización de la industria se genera una brecha en países periféricos o dependientes con fuerzas de trabajo de salarios bajos y de gran disponibilidad de desocupados, o de un ejército de reserva amplio, disponible para la incorporación barata al mercado de trabajo (Montané \& Sartorio, 2018). Este escenario genera falta de talento, pues se prevé que cerca de la mitad de la fuerza laboral deberá ser re-capacitada o reemplazada en los próximos cinco años (McKinsey Global Institute, 2019b). 
Y aunque hay quienes dicen que la automatización masiva es el precio que se ha de pagar por la prosperidad productiva, esto no se corrobora, de hecho, las tecnologías han avanzado rápidamente, pero las industrias que más las usan no han logrado mejorar el crecimiento de la productividad total de los factores, el producto o el empleo (Acemoğlu, 2021). Baldwin (2019) menciona que estas computadoras pensantes no son potenciadores de la productividad laboral, sino están diseñadas para reemplazar a los trabajadores al ser mucho más baratas. Las estimaciones del desplazamiento laboral oscilan entre uno de cada diez empleos, lo que significa millones de puestos de trabajo, todo esto con la consiguiente agitación (Baldwin, 2019). Por lo que se puede decir que la relación entre automatización, niveles de empleo y de desempleo es compleja, pues si bien se habla de los efectos del desempleo tecnológico, por otro lado, surge la polarización del mercado de trabajo que deviene en la reducción de los empleos de cualificación media rutinarios lo que acompañado de otras grandes tendencias mundiales como la globalización y el cambio demográfico tendiente al envejecimiento, acentúan la complejidad de la relación y hacen previsible que las tareas que implican la resolución de problemas, que son difíciles de codificar, vayan ganando en importancia relativa respecto a las tareas rutinarias (Arguello Verbanaz, 2019). Por ejemplo, vemos que el potencial para automatización varía según el sector y la actividad de trabajo específica; según los datos de la Oficina de Estadísticas laborales de EEUU los 3 sectores con más potencial de automatización son: el alojamiento y alimentación $75 \%$, el sector de la fabricación con el $60 \%$ y agricultura con el $60 \%$, mientras que los sectores con menos potencial de automatización son: la salud y asistencia social con 36\%, servicios profesionales con el $35 \%$ y los sectores educativos con el 27\% (Chui et al., 2017). En definitiva, se proyectan inconvenientes, pues como vemos la globalización y la automatización a menudo favorecen a los que ya son favorecidos, generando más desigualdad (Baldwin, 2019) y agravando las pérdidas de empleos y trastornos económicos (Acemoğlu, 2021).

Además, de los cambios en el desarrollo tecnológico, en la década del setenta, el crecimiento sostenido por los pilares del Estado de Bienestar se detiene y se produce una crisis significativa en la que desaparece el trabajo indefinido y aparece la flexibilidad laboral y se modifican las relaciones contractuales (Olmos \& Silva, 2011). De modo que las condiciones de empleo y la pertenencia a colectivos cambian e inciden en el mercado de trabajo. Cambios como: la precariedad laboral, la inseguridad en la relación laboral, el empleo temporal o eventual, la insuficiencia en los ingresos salariales que determinan el nivel de vida, la autonomía y la posición social incrementan la incertidumbre y la inseguridad respecto al futuro sociolaboral (Rubio Arribas, 2009). En América Latina en 2006 existían más de 23 millones de personas en condición de desempleo abierto y 
aproximadamente 103 millones trabajaban en el sector informal, muchas veces sin derechos laborales ni protección social, además era visible un déficit de empleo formal de 126 millones de trabajadores, siendo ese déficit más acentuado en el caso de las mujeres y los jóvenes (Organización Internacional del Trabajo, 2006). En 2019 la mayoría de las personas trabajadoras en Brasil, Colombia y México consideraban la actividad autónoma como la principal alternativa cuando no es posible encontrar trabajo asalariado, de hecho, el 70 por ciento de los participantes afirmó que el trabajo autónomo temporario o informal es una opción viable en épocas de incertidumbre económica (McKinsey Global Institute, 2019a). Con la crisis de la COVID-19, se han acentuado los problemas estructurales preexistentes y los déficits de trabajo decente en la región (Organización Internacional del Trabajo, 2021).

Sumado a estos cambios, los sindicatos se debilitan (Milanovic, 2020), pues antes la condición salarial estaba ligada a la pertenencia a colectivos y su consiguiente garantía de protección (Castel, 2010). En la actualidad la afiliación sindical en todo el mundo ha ido disminuyendo con el tiempo, a pesar de que existen algunos puntos brillantes en determinados países latinoamericanos donde la afiliación aumentó. Esto se debe al paso de los empleos manufactureros a los de servicios, la externalización de los empleos sindicalizados, la informalización de la economía y la mutación de la relación laboral o la automatización y las restricciones legales (McKinsey Global Institute, 2021a). Por tanto, la descolectivización o reindividualización, transforma el colectivo de trabajo, que incluso puede ser completamente disuelto, sobre todo en modalidades más avanzadas del "trabajo inmaterial" (Castel, 2010), incluso en el trabajo considerado material pues si bien se entiende de acuerdo con Hardt y Negri (2000) que el trabajo inmaterial se basa en las capacidades intelectuales y afectivas del trabajador, en la actualidad el trabajo flexible y remoto han difuminado de manera gradual los límites del trabajo material e inmaterial (en Cortés Díaz et al., 2020). Según datos de la Organización Internacional del trabajo en América Latina el porcentaje de declinación de afiliación sindical paso del 16\% en el 2000 al 15\% en el 2016, esto es especialmente importante considerar pues la COVID-19 ha desafiado a todos los actores involucrados en el sistema de protección social a (re)considerar sus papeles y responsabilidades (McKinsey Global Institute, 2021a). Por tanto, se puede decir que el cambio tecnológico hizo que los trabajadores no calificados de las fábricas fueran más reemplazables, mientras que la pertenencia sindical hizo que los trabajadores pierdan el sentido de un futuro asegurado (Baldwin, 2019).

Entonces, los cambios en el mercado laboral como parte del tránsito del capitalismo industrial al capitalismo postindustrial tienen implicaciones en los proyectos y las prácticas cotidianas de los sujetos (Castel, 2010) y más aún esta siguiente fase de 
automatización que se define como más disruptiva que las anteriores (Bitar, 2014). Por lo tanto, si en el capitalismo industrial el trabajo era el elemento clave para la cohesión social, posteriormente, el trabajo sigue siendo central, pero el escenario de progreso prometido parece haberse detenido, el trabajo ya no representa progreso sino incertidumbre, se marcan nuevos riesgos: la precarización de las relaciones de trabajo, la descolectivización y la ubicua exhortación a afianzar nuestro individualismo, sin considerar las falencias del mercado laboral. Es ahora cuando el proletariado se convierte en asalariado que "tiene mucho más que perder que sus cadenas" y se pone en evidencia el riesgo de "quedarse afuera de la sociedad salarial" (Castel, 2010).

\section{Respuestas desde el Estado que generan más incertidumbre}

Stiglitz (2003) dice que históricamente se han configurado diferentes puntos de vista sobre el papel del Estado: por un lado en los siglos XVIII Y XIX los economistas franceses postularon que el Estado debía fomentar activamente el comercio y la industria, los denominados mercantilistas. Frente a ellos Adam Smith defendió la idea de que el Estado debía desempeñar un papel limitado; la influencia de las ideas de Smith devienen en la doctrina conocida con el nombre de laissez jaire, en la que el Estado debía dejar hacer al sector privado y no intentar regularlo. Aunque esta idea fue aceptada por muchos círculos de académicos, no fue aceptada por todos, entre esos Marx, quien defendió la idea de que el Estado debía intervenir más en el control de los medios de producción (Stiglitz, 2003). Como vemos el debate de libre mercado frente al intervencionismo estatal no es nuevo pero sigue vigente. Uno de los hitos clave en el debate fue la Gran Depresión que sacó a la palestra problemas que existan desde hacía tiempo y se llegó al convencimiento de que los mercados habían fallado y que era necesario que el Estado hiciera algo, el defensor de estas ideas fue Keynes (Stiglitz, 2003).

Las ideas keynesianas luego de la Segunda Guerra Mundial dan pie, entre otras cosas, al Plan Marshall concebido como una nueva forma de organización política y manejo económico, donde la mayoría de los gobiernos asumen que no es posible mantener una forma de organización basada en la desregulación económica propugnada por el liberalismo. Por lo que en adelante, el Estado interviene entregando servicios y beneficios, pero otorgando además una serie de mecanismos y recursos que introducen certidumbre y seguridad, de modo que en la denominada "Edad de Oro" el crecimiento 
económico de las sociedades industriales consiguen la ecuación keynesina de compaginar la justicia social con la acumulación de capital (Olmos \& Silva, 2011). Sin embargo, a partir de los años setenta disminuye el crecimiento económico sostenido y el pleno empleo que se había logrado en la mayor parte de los países occidentales desde los años cincuenta ya no existe. Con esto comienza un proceso de lo que Olmos y Silva (2011) denominan un desbaratamiento del Estado de Bienestar Keynesiano frente a lo cual diversos sectores proponen desmantelar las instituciones de protección social, disminuir el gasto público, bajar los impuestos y retomar un camino hacia un desarrollo de la economía autorregulada. Esta propuesta encabezada por Hayek y Friedman ubica al Estado como un obstáculo para la libre economía (Olmos \& Silva, 2011). Entonces, se sitúan por un lado las ideas de Keynes el arquitecto intelectual del New Deal y del moderno Estado del Bienestar que había puesto en evidencia el hundimiento del mercado y la necesidad de la intervención estatal y, por otro lado, las ideas de Friedman quien luchó contra los que creían que los gobiernos tienen la responsabilidad de intervenir en el mercado para suavizar su dureza (Klein \& García, 2007).

Esta forma de capitalismo que concentra la gran mayoría de la producción en el sector privado, sin protagonismo del Estado, Milanovic (2020) la denomina capitalismo meritocrático liberal, donde a diferencia del capitalismo clásico y del capitalismo socialdemócrata que definió a los Estados de Bienestar, las personas tienden a ser ricas en capital y mano de obra, es decir, generan sus ingresos tanto de inversiones como de trabajo, lo que sumado a la globalización cambia la naturaleza del trabajo industrial y la formación de sindicatos (Milanovic, 2020). Por otro lado, Naoimi Klein explica el desmantelamiento de los restos del Estado de Bienestar con el denominado "capitalismo del desastre", pregonado por Friedman, cuyo objetivo es promover a nivel global el modelo de desarrollo neoliberal, lo que quiere decir, que se concibe a las fuerzas económicas del mercado como la oferta y la demanda, la inflación, el desempleo, como fuerzas naturales e inmutables. Por tanto, se hace necesario borrar todo rastro de intervención estatal que impida la consecución del libre mercado. En este sentido, el capitalismo del desastre "aprovecha" el trauma colectivo para promover la eliminación del rol público del Estado, la absoluta libertad de movimientos de las empresas privadas y un gasto social prácticamente nulo (Lange $V, 2010$ ).

A partir de 1989 una ideología absolutamente opuesta a las antiguas concepciones keynesianas, fue asumida como modelo de desarrollo económico predominante y hegemónico a nivel mundial, el neoliberalismo. "El fin de la historia" de Fukuyama, representa el paso del neoliberalismo a ser el único pensamiento posible 
(Olmos \& Silva, 2011). De modo que, el sistema económico capitalista neoliberal y su lógica se impregna en todas las esferas de la vida, lo que determina que se equipare capitalismo con realidad ahogando automáticamente cualquier afuera posible (Montenegro \& Pujol, 2013). Según Laval y Dardot se fija al neoliberalismo como una nueva racionalidad política, retomando la hipótesis foucaultiana, que determina una lógica normativa generalizada, desde el Estado hasta lo más íntimo de la subjetividad (Wegelin, 2021). Esto significa que se propagan ideas a favor de los mercados y en contra de la intervención estatal (Bitar, 2014). Una de las ideas es la representada por Hayek quien decía que en el preciso instante en el que el Estado legisle simplemente para corregir o rectificar algún resultado producido por la "espontaneidad de mercado", devendrá en sistema político tiránico o totalitario (Blanco, 2018). Bajo esta lógica Hayek advierte que el desarrollo del Estado de Bienestar puede poner en riesgo la libertad individual y la democracia (Acemoğlu, 2021).

Pero, a la larga, la preocupación de Hayek resulto errónea, pues ni la libertad ni la democracia sufrieron en el Reino Unido o en los países escandinavos que adoptaron similares programas de bienestar (Acemoğlu, 2021). Pues esta idea del predominio de los mercados dice Stiglitz et al. (2021) descuida la historia de cómo surgieron los mercados, pues la mano invisible del mercado dependía de la mano más dura del Estado. De hecho, el libre mercado y sus promesas han sido cuestionados en diferentes momentos de la historia, como vimos en la Gran Depresión que impulsó a que el Estado cumpla un rol protagónico (Rosales, 2020). Durante los últimos años la lógica neoliberal ha vuelto a ser cuestionada por su incapacidad de superar la pobreza, la exclusión y por aumentar la desigualdad social, por ejemplo: en la crisis económica mundial 2008 - 2010, en la que se puso de manifiesto que los Estados siguen siendo los garantes del funcionamiento de la economía de mercado (Olmos \& Silva, 2011). Entonces, a pesar de que la lógica del capitalismo del desastre espera a que se produzca una crisis de primer orden o estado de shock para luego vender al mejor postor los pedazos de la red estatal a los agentes privados (Klein \& García, 2007), el Estado sigue siendo quien provee bienestar para que la población pueda participar en el mercado y su intervención no representa el comienzo de una era distópica de gobierno opresivo, pues ningún mercado exitoso puede sobrevivir sin los cimientos de un Estado sólido y funcional (Stiglitz et al., 2021).

Ahora, si bien se ha apuntado a la importancia de la intervención del Estado, así como los mercados suelen fallar el Estado también falla. Stiglitz habla de cuatro causas de la incapacidad sistemática del Estado: su reducida información, su reducido conocimiento de las respuestas privadas, su reducido control de la burocracia y las limitaciones que imponen los procesos políticos. Lo anterior seria para Friedman, 
suficiente para que el Estado se abstenga de intentar resolver las deficiencias del mercado (Stiglitz, 2003). Sin embargo, en un contexto de economías mixtas el Estado actúa, aunque falle. Pero ¿qué pasa cuando el Estado falla? o peor aun cuando pasa a ser un Estado reparador que solo actúa después de una falla explícita del mercado y cuando lo hace sus políticas son escasas y tardías (Mazzucato, 2020).

Si consideramos los regímenes de bienestar de Esping-Andersen (1993) que se estructuran a través de las diferentes formas de distribución de la responsabilidad en la provisión de bienestar desde las esferas del Estado, el mercado y las familias; inicialmente se asume que el Estado responderá a esta necesidad, pero como vemos las ideas y prácticas del neoliberalismo se esforzaron en hacer retroceder las garantías existentes a la protección social desde el Estado (Jenson, 2010). De modo que, el Estado retrocede y da un papel más amplio para las esferas del mercado, familias y comunidades para que provean bienestar, sin embargo, la lógica del mercado no contempla en sus objetivos la igualdad, ni la distribución equitativa o la sobrecarga de las esferas familiar y comunitaria, y esto genera más pobreza y problemas sociales (Jenson, 2010).

El retroceso del Estado y los nuevos riesgos que surgen en el ámbito laboral ponen en entredicho la propia estructura del Estado y se asume cierta incapacidad del sistema de hacerse cargo de estos riesgos, es decir, la proliferación de los riesgos contribuye al cuestionamiento de las capacidades del Estado social (Castel, 2010), con la consiguiente incertidumbre que deviene de la dependencia al mercado en un sistema que aboga por la igualdad de oportunidades cuando no se ha alcanzado la igualdad de posiciones (Dubet, 2011). Pues cuando la esfera estatal ya no constituye una certeza y los riesgos recaen diferencialmente a nivel individual, incluso regional. En América Latina, se habla de un Estado Residual que provee bienestar solo cuando las esferas del mercado y la familia fallan (Ubasart-González \& Minteguiaga, 2017). Salvia (2007) menciona que con modelos de desarrollo desigual, la degradación de la sociedad salarial se manifiesta en la desestabilización de los empleos estables, la instalación de la precariedad como destino, que consiste en vivir al día a través de un trabajo no registrado y la aparición de un nuevo perfil social al que se denomina como supernumerarios, sujetos que el capital descalifica en el plano económico, cívico y político, una masa marginal "no funcional” (Salvia, 2007). Entonces, el Estado exige a los trabajadores hacerse cargo ellos mismos de su propio recorrido profesional, se les exhorta a ser un individuo (Castel, 2010).

Lo anterior determina una respuesta pasiva del Estado frente a los cambios en el mundo del trabajo y el aumento del desempleo (Brown, 2016). Es aquí cuando surgen los programas "productivistas" y orientados a la inversión, es decir, programas sociales que buscan promover la participación económica y generar tasas de rendimiento positivas 
para la economía (Jenson, 2010). Este enfoque articula la palabra trabajo (work) con bienestar (welfare) (Brown, 2016), definiendo lo que se debe hacer, tener y ser para acceder a un mínimo de bienestar. Por tanto, se asume una nueva visión de la política económica y del desempleo, que deja de estar ligada a variables macroeconómicas, pues se asume que existen suficientes oportunidades de empleo, por lo que la problemática recae en los individuos; entonces se pasa de definir el desempleo como un fenómeno social a definirlo como un problema individual, surgen así "los sin trabajo". Por esto, la lógica de las políticas de activación está orientada a mejorar los niveles de integración social y económica de las personas desocupadas a partir de su (re) inserción al mercado laboral, y el empleo resulta el mecanismo básico de inclusión social, pero también se incrementan las restricciones y condiciones hacia las personas beneficiarias de la política asistencial, para fomentar su "activación", es decir, que reingresen al mercado laboral lo más rápido posible (Brown, 2016).

Los procesos productivos y la organización del trabajo implican una dimensión política, donde la explotación se encuentra entrecruzada por relaciones de dominación (Giavedoni, 2014). En el paradigma de activación uno de los inconvenientes que se pueden analizar es la definición de los problemas de desigualdad desde la responsabilidad individual y específicamente desde las características del individuo que le permitan insertarse laboralmente (Brown, 2016). Entonces la teoría del capital humano, en la lógica neoliberal, define al sujeto por las capacidades productivas, por acumulación de conocimientos generales o específicos, actitudes, destrezas, de modo que induce a los individuos a considerarse empresarios de sí mismos (Martinez, 2014). Entonces, se plantea que la desigualdad de los ingresos está positivamente correlacionada con la desigualdad en la educación y con otras formas de aprendizaje, por esto la educación se ve como una inversión en el capital humano para elevar y conservar la productividad y la empleabilidad. Con esto no se cuestionan las potencialidades que tiene una economía en la generación de puestos de trabajo y tampoco variables de desigualdades estructurales como: el sexo, la raza, el entorno social y la clase social. Esto nos permite analizar cómo pese a que según la OIT en América Latina la tasa promedio de desempleo urbano para la región bajó hasta un 6,4\%, lo que parece ser un logro no advierte la existencia de 15 millones de personas en la región que carecen de trabajo y según Giavedoni (2014) aquí es donde los matices importan, pues no se considera el trabajo precarizado, en negro, informal, el trabajo independiente que adolece de gran informalidad y carece de protección y seguro social, condiciones que en esta lógica pulverizan al sujeto sin considerar el sistema (Giavedoni, 2014). En definitiva, la demanda/oferta de trabajo y la calidad de los empleos creados no son incluidos en el análisis del desempleo, mientras 
que se hace un énfasis en las características de los individuos lo que redunda en una mayor culpabilización y responsabilización individual por problemas que, como la desocupación y la pobreza, son de carácter social (Brown, 2016).

Brown (2016) indica que como estos modelos equiparan la inclusión social con empleo las políticas sociales de contraprestaciones son de bajas cuantías y de limitada duración en el tiempo, para que se mantenga latente la necesidad del trabajo. Una vez más se asume al individuo como incapaz de manejar los recursos y que bajo los principios del cálculo racional, en el que la propensión al ocio resulta un factor relevante, opta de forma voluntaria permanecer en el sistema de asistencia. De forma que, la lógica workfare debe comprenderse como el triunfo de los principios de obligación social sobre los de derechos sociales y como un retroceso en materia de responsabilidad pública, pues individualiza cada vez más el diagnóstico sobre la problemática del desempleo y sobre las contraprestaciones necesarias para su superación. Lo que representa un inconveniente pues no permite abordar la problemática desde una perspectiva macrosocial ni encontrar sus causas sistémicas (Brown, 2016). Siguiendo a Dubet (2011) esta lógica responde al modelo de oportunidades donde el contrato social se individualiza, por tanto, se definen a las oportunidades como responsabilidades de los individuos y éstos deben ser activos y movilizarse para merecer la ayuda, bajo esa lógica el subsidio se "trueca" a cambio de responsabilidades y de compromisos (Dubet, 2011). Esto por un lado genera sujetos que pueden ajustarse, por sus posiciones y por ser hipercompetitivos, y otros que igualmente comprometidos en el cambio, son impotentes para dominarlo pues carecen de las condiciones requeridas para hacer frente. Este segundo grupo, los denominados individuos por defecto, ponen en evidencia la incapacidad de ser más competitivos, cuando no pueden escapar al miedo de ser anulados del mercado de trabajo de la noche a la mañana (Castel, 2010). En estos individuos se cristaliza la incertidumbre y el miedo, que definen su biografía sin considerar las desventajas estructurales y su efecto diferencial, heterogéneo y al mismo tiempo más focalizado, creando nuevas situaciones de riesgo, cristalizando "biografías de riesgo" (Saraví, 2007).

\section{Conclusiones para gestionar la incertidumbre desde el Estado}

La provisión de certeza y de bienestar está mediada por la interacción de las esferas de Bienestar: el Estado, el mercado y la familia (Esping-Andersen, 1993). Por tanto, las respuestas desde el Estado que apuntan al universalismo como patrón de relaciones sociales, de reconocimiento y de solidaridad, constituyen una propuesta de organización 
de la protección y una matriz de redistribución (Danani, 2017). Pues, como dicen Van Parijs \& Vanderborght (2017) los Estados de bienestar "pasivos" que sostienen programas de ingreso mínimo sujetos a la comprobación de recursos, centran sus prestaciones en quienes están inactivos y en consecuencia los mantienen inactivos, no representan un camino para menguar esas incertidumbres. Sumado a esto, en América Latina con un Estado históricamente frágil, donde las propuestas de intervención están atravesadas por la lógica de que a menores recursos disponibles se requiere llegar a los sectores que tengan "verdadera" necesidad, se determina un principio focalizador en el eje ordenador de las instituciones y de la vida social, que deja de lado políticas universalistas definidas como un despilfarro (Danani, 2017).

Considero como propuesta de solución desde el Estado para contrarrestar las incertidumbres y reivindicar el rol protector frente a estos riesgos, el ingreso básico universal (Van Parijs \& Vanderborght, 2017), pues permite restablecer el equilibrio entre la responsabilidad personal y la solidaridad nacional, ir más allá del mito de una seguridad absoluta e instaurar una deontología del riesgo, para gestionarlos desde la protección social. De esta forma, se pueden analizar los recursos con los que cuenta el Estado para no adicionar miedo a los sujetos (Van Parijs \& Vanderborght, 2017), pues si las reglas del juego del capitalismo cambiaron, deberían cambiar también las de la protección y de seguridad social (Castel, 2010). Pues como dicen Van Parijs \& Vanderborght (2017) frente a esta reconfiguración del mundo que da lugar a amenazas sin precedentes, deben surgir oportunidades sin precedentes. Con esto se plantea el ingreso básico universal, que si bien se deberá adaptar a los diferentes contextos se sontiene en la imposición fiscal, es decir, en un impuesto de renta progresivo para que el sistema se pueda financiar y pueda pagar un ingreso regular y en efectivo a todos los miembros de una sociedad, sin importar los ingresos que obtengan de otras fuentes y sin ponerles ninguna condición. Lo anterior cuestiona la lógica individualista porque requiere considerar una libertad para todos y materializa una inversión en la lógica del rol del Estado, pues esta propuesta se sostiene desde un "Estado de bienestar activo", que cubra nuestras necesidades de salud, educación, protección social y nos permita tener un piso firme en el que podamos ponernos de pie como individuos libres de la dependencia al mercado, con esperanza y así calmar nuestras ansiedades, incertidumbres y fortalecer nuestras comunidades (Van Parijs \& Vanderborght, 2017). De modo que, si bien se ha cuestionado la sostenibilidad del ingreso básico y su viabilidad en mediano plazo sobre todo en Latinoamérica y en la Argentina, la pandemia ha puesto en evidencia la necesidad de repensar el modelo de desarrollo a través de un nuevo pacto social y fiscal (CEPAL, 2020). Esto sin duda, 
además de requerir la consideración del contexto requiere desafiar la lógica neoliberal impregnada en la subjetividad y pensar en la posibilidad de un afuera del capitalismo.

Como se analizó en secciones anteriores cuando el mercado constituye una fuente de incertidumbre y desde el Estado las respuestas son insuficientes se pone el peso en la esfera de la comunidad y la familia. Sobre todo, en Latinoamérica, con estrategias aunque limitadas y defensivas, propongo considerar como otro ejemplo de respuesta frente a las incertidumbres la denominada economía social, como desafío a la lógica neoliberal. Presta (2017) plantea que la economía social y solidaria que se consolida en Latinoamérica en el noventa constituye una posibilidad de resignificación de los sentidos del trabajo que apela a la potencialidad del ser humano como fundamento del gobierno de sí transformando las relaciones entre saber, poder y gubernamentalidad (Presta, 2017). Como indica Salvia (2007), una vez debilitado el ideal de progreso, la crisis provocada por el desempleo estructural, la precarización laboral, la pobreza y la desafiliación institucional obligan a los sujetos a generar estrategias alternativas de subsistencia colectiva. Estas alternativas defensivas, una vez más, se encuentran en la autogestión familiar, los emprendimientos vecinales, las cooperativas populares y otras iniciativas donde prevalece el fin social sobre el lucro individual. Entonces la economía social, por un lado, busca generar un abaratamiento de los costos sociales y familiares de reproducción de la fuerza de trabajo y, por otra parte, permitirles a los trabajadores escapar parcialmente del mercado de trabajo y de las relaciones salariales , lo que implica en un sentido amplio las posibilidades de desmercantilización (Salvia, 2007). Por tanto, el sujeto en el dispositivo de la economía social y solidaria se define como emprendedor, solidario y propende a la realización del trabajo humano no alienante, lo cual define específicos esquemas de percepción, saberes y prácticas diferentes a las del neoliberalismo, con lo cual se puede decir que nos encontramos con este sujeto-enresistencia (Presta, 2017). Sin embargo, aunque el Banco Mundial exhorta a generar emprendimientos productivos de pequeña escala orientados al autoconsumo o a la comercialización en circuitos locales (Presta, 2017), la economía social y solidaria se ve cuestionada en su carácter emancipador al aún ser local, por lo que no logra esta independencia del mercado más que para la subsistencia, ni garantiza una reparación de los lazos de integración y de los soportes perdidos por el desmantelamiento de los vínculos asociativos del trabajo asalariado (Salvia, 2007).

Como conclusión puedo decir que el Estado y las políticas sociales son interpelados como certeza frente al miedo, como categoría emocional, económica y política. Castel (2010) dice que debemos asumir la incertidumbre, pero teniendo en cuenta que el futuro dependerá en gran medida de lo que hagamos o dejemos de hacer hoy. Entonces ¿qué 
debe hacer el Estado hoy?, cuando es evidente que los riesgos se han generalizado, cuando el trabajo deviene incertidumbre, cuando las políticas focalizadas generan más incertidumbre (Danani, 2017) y cuando el modelo de oportunidades individualiza y produce subjetividad desprovista de dignidad (Dubet, 2011). Desde mi perspectiva el Estado debe asumir respuestas imposibles frente a riesgos que se consideraban imposibles años atrás, siguiendo a Derrida, cuando seguimos los posibles, vamos por ese camino una y otra vez, entonces, sólo lo imposible, desde el cual y hacia el cual se abre la deconstrucción, nos permite pensar otro modo de ser (Chun, 2012); sería entonces esa deconstrucción de lo posible en las políticas sociales una apertura a un porvenir donde el miedo no sobrepase a la esperanza; solo así el ingreso universal, por ejemplo, dejaría de ser una utopía .

\section{Referencias}

Acemoğlu, D. (2021). Para revertir el aumento de la desigualdad hay que controlar estrictamente la automatización. Finanzas \& Desarrollo, 6.

Arguello Verbanaz, S. (2019). Los efectos de la automatización sobre el trabajo. Biblioteca Congreso Nacional de Chile. https://obtienearchivo.bcn.cl/obtienearchivo?id=repositorio/10221/26941/1/Lo s_efectos_de_la_automatizacion_sobre_el_trabajo.pdf

Baldwin, R. (2019). The globotics upheaval: Globalization, robotics, and the future of work. Oxford University Press.

Bitar, S. (2014). Las tendencias mundiales y el futuro de América Latina. CEPAL.

Blanco, J. P. (2018). Isaiah Berlin, Friedrich Hayek y Milton Friedman pasean por villa miseria. Los límites de la concepción liberal de la libertad1. Tabula Rasa, 29, 183201.

Brown, B. (2016). Sistema de Protección social y Programas de Transferencias Monetarias Condicionadas. El “paradigma de activación” en Argentina 2003-2013. Documentos de Trabajo CIEPP, 99.

Castel, R. (2010). El ascenso de las incertidumbres. Trabajo, protecciones, estatuto del.

CEPAL. (2020, mayo 12). CEPAL propone avanzar hacia un ingreso básico para ayudar a la población más vulnerable a superar los efectos del coronavirus [Text]. CEPAL. https://www.cepal.org/es/comunicados/cepal-propone-avanzar-un-ingresobasico-ayudar-la-poblacion-mas-vulnerable-superar 
Chui, M., George, K., Manyka, J., \& Miremadi, M. (2017). Hombre máquina: Una nueva era de automatización en manufactura. Mckinsey. https://www.mckinsey.com/business-functions/operations/ourinsights/human-plus-machine-a-new-era-of-automation-in-manufacturing/esES

Chun, S. (2012). El porvenir de Jacques Derrida. Contrastes. Revista internacional de filosofia, 17.

Cortés Díaz, G., Henao Godoy, N., \& Osorio Linero, V. (2020). Trabajo remoto en tiempos de covid-19 y su impacto en el trabajador.

Danani, C. (2017). Políticas sociales universales: Una buena idea sin sujeto Consideraciones sobre la pobreza y las políticas sociales. Revista Sociedad, 37.

Dubet, F. (2011). Repensar la justicia social: Contra el mito de la igualdad de oportunidades. Siglo Veintiuno Editores Argentina.

Esping-Andersen, G. (1993). Las tres economías políticas del Estado de bienestar. En: Desarrollo Institucional y Conflicto. En De la geopolítica a la distribución del ingreso. Serie Estado, Gestión Pública y Desarrollo. CAF.

Giavedoni, J. G. (2014). Trabajador asalariado y trabajador flexigurado. Transformaciones de la subjetividad en torno al trabajo. Melisa Campana y José Giavedoni (comps.), Debates sobre Estado, Gobierno y Control Social. Gobernando las desigualdades, Rosario, E-Book.

Hewitt, J., \& Monge-González, R. (2018). La automatización en el sector de los servicios offshore: Impactos sobre la competitividad y la generación de empleo.

Jenson, J. (2010). Diffusing ideas for after neoliberalism: The social investment perspective in Europe and Latin America. Global Social Policy, 10(1), 59-84.

Klein, N., \& García, I. F. (2007). La doctrina del shock: El auge del capitalismo del desastre (Vol. 1). Paidós Barcelona.

Lange V, C. (2010). La Doctrina del Shock. El auge del capitalismo del desastre. Revista INVI, 25(70), 225-227. https://doi.org/10.4067/S0718-83582010000300007

Martinez, L. (2014). Tecnologías de gobierno y neoliberalismo en la actualidad: Lo humano como capital, el individuo como empresa. Cap VII Pag. 131-150. En, Debates sobre Estado, Gobierno y control social. Gobernando las desigualdades. Organizadores Campana M y Giavedoni J G.

Mazzucato, M. (2020, diciembre 27). Capitalism After the Pandemic. https://www.foreignaffairs.com/articles/united-states/2020-10-02/capitalismafter-covid-19-pandemic 
McKinsey Global Institute. (2019a). Latinoamérica y sus "missing middles": Un grupo dinámico de empresas medianas y el poder adquisitivo de la clase media. https://www.mckinsey.com/featured-insights/americas/latin-americas-missingmiddle-of-midsize-firms-and-middle-class-spending-power/es-cl

McKinsey Global Institute. (2019b, enero 15). Building a creative talent strategy. https://www.mckinsey.com/business-functions/organization/our-insights/arewe-long-or-short-on-talent/es-CL

McKinsey Global Institute. (2021a). La transición de los sindicatos: ¿qué papel tendrán en el futuro del trabajo? https://www.ilo.org/infostories/es-ES/Stories/LabourRelations/trade-unions\#revitalization

McKinsey Global Institute. (2021b). El futuro del trabajo después del COVID-19. https://www.mckinsey.com/featured-insights/future-of-work/the-future-ofwork-after-covid-19

Milanovic, B. (2020). Choque de capitalismos. Foreign Affairs Latinoamérica, 20(3), 125-136.

Montané, F. M., \& Sartorio, L. (2018). Tecnología y generación de empleo en el siglo XXI; desafíos y propuestas. Academia Nacional de Ciencias Morales y Políticas.

Montenegro, M., \& Pujol, J. (2013). La fábrica de conocimientos: In/corporación del capitalismo cognitivo en el contexto universitario. Athenea Digital. Revista de pensamiento e investigación social, 13(1), 139-154.

Olmos, C., \& Silva, R. (2011). El desarrollo del Estado de bienestar en los paÃses capitalistas avanzados: Un enfoque socio-hist $\tilde{A} \$ 3 \$$ rico. Revista Sociedad y Equidad, 1.

Organización Internacional del Trabajo. (2006). Trabajo decente en las Américas: Una agenda hemisférica, 2006-2015. https://www.ilo.org/public/spanish/standards/relm/rgmeet/americas.htm

Organización Internacional del Trabajo. (2021). Perspectivas Sociales y del Empleo en el Mundo Tendencias 2021 (Primera). Unidad de Producción de Pubcliaciones. https://www.ilo.org/wcmsp5/groups/public/---dgreports/---dcomm/--publ/documents/publication/wcms_825200.pdf

Presta, S. R. (2017). Economía social y solidaria y conflicto social. Consideraciones sobre las formas de gobierno de la fuerza de trabajo en el umbral de nuevos cambios socio-técnicos.

Rosales, O. (2020). Después de la pandemia: Tendencias globales probables. Instituto Igualdad. https://institutoigualdad.cl/2020/05/07/despues-de-la-pandemiatendencias-globales-probables/ 
Rubio Arribas, F. J. R. (2009). Aspectos sociológicos del desempleo/paro y de la precariedad laboral. Nómadas. Critical Journal of Social and Juridical Sciences, 24(4).

Salvia, A. (2007). Consideraciones sobre la transición a la modernidad, la exclusión social y la marginalidad económica. Un campo abierto a la investigación social y al debate político. En Sombras de una marginalidad fragmentada. Aproximaciones a la metamorfosis de los sectores populares de la Argentina. Buenos Aires (Argentina): Miño y Davila.

Saraví, G. (2007). Nuevas realidades y nuevos enfoques: Exclusión social en América Latina. De la pobreza a la exclusión. Continuidades y rupturas de la cuestión social en América Latina. Buenos Aires, CIESAS \& Prometeo.

Smith, N. (2021, abril). Bidenomics, explained. https://noahpinion.substack.com/p/bidenomics-explained

Stiglitz, J. E. (2003). La economía del sector público (Vol. 24). Antoni Bosch Editor.

Stiglitz, J., Tucker, T., \& Zucman, G. (2021). El Estado hambriento. https://nuevospapeles.com/

Ubasart-González, G., \& Minteguiaga, A. (2017). Esping-Andersen en América Latina: El estudio de los regímenes de bienestar. Política y gobierno, 24(1), 213-236.

Ubieto, G. (2021). El covid acelera la automatización en las empresas y amenaza 4,6 millones de empleos en España. elperiodico. https://www.elperiodico.com/es/economia/20210219/covid-aceleraautomatizacion-empresas-amenaza-11531897

Van Parijs, P., \& Vanderborght, Y. (2017). Ingreso básico: Una propuesta para una sociedad libre y una economía sensata. Grano de Sal.

Wegelin, L. (2021). Ideología o racionalidad? Interrogaciones epistemológicas sobre la relación neoliberalismo-democracia a partir de la perspectiva foucaultiana. https://dx.doi.org/10.5209/poso.71154 